\title{
Características Antropométricas de Futbolistas Profesionales Chilenos
}

\author{
Anthropometric Characteristics of Chilean Professional Football Players
}

\author{
Carlos Jorquera Aguilera*; Fernando Rodríguez Rodríguez**; María Ignacia Torrealba Vieira*; \\ José Campos Serrano*; Natalia Gracia Leiva* \& Francis Holway ${ }^{* * *}$
}

\begin{abstract}
JORQUERA, A. C.; RODRÍGUEZ, R. F.; TORREALBA, V. M. I.; CAMPOS, S. J.; GRACIA, L. N. \& HOLWAY, F. Características antropométricas de futbolistas profesionales chilenos. Int. J. Morphol., 31(2):609-614, 2013.

RESUMEN: La tendencia en la formación en el futbol, determina que las variables antropométricas sean importantes al momento de definir entre otras cosas, la posición de juego más idónea. Estos parámetros morfológicos son una parte esencial de la evaluación y selección de los deportistas. Estos patrones permiten determinar la evolución física del futbolista desde temprana edad, lo que ayuda enormemente en la intervención de los mismos. Se evaluó antropométricamente, a un total de 406 futbolistas profesionales, pertenecientes a 15 clubes chilenos, de los cuales 12 pertenecen a Primera División y 3 clubes a Primera B. Existen diferencias en algunas variables antropométricas básicas por posición de juego, que nos ponen en desventaja frente a otros jugadores internacionales, como la estatura. No hay diferencias en el Phantom ni en el somatotipo por posición de juego, es decir tienen variables relativamente homogéneas. Esto podría hacernos pensar que un jugador puede desempeñarse en cualquier posición de juego, pero hemos visto que la estatura, el peso y la masa grasa son distintas por cada posición, por lo que cada jugador tiene su función y sus propias características en las que los entrenadores y preparadores deben fijarse al momento de ubicar a cada jugador en su función específica.
\end{abstract}

PALABRAS CLAVE: Antropometría; Futbol; Posición de juego.

\section{INTRODUCCIÓN}

El fútbol es el deporte más popular del mundo y en nuestro país el más practicado de manera recreativa y/o amateur. El futbol es popular debido a que no requiere de cualidades o capacidades físicas desarrolladas al máximo, ya que depende de una gran variedad de factores. Stolen et al. (2005), señala que los Jugadores profesionales de futbol pueden tener un consumo máximo de oxígeno entre $50-75 \mathrm{~mL} / \mathrm{kg}^{-1} / \mathrm{min}$, lo que es razonable en comparación a otros deportes.

La tendencia en la formación en el futbol, determina que las variables antropométricas sean importantes al momento de definir entre otras cosas, la posición de juego más idónea, determinando el rendimiento en un salto o en un sprint (Dey et al., 2010).

En la actualidad, un deportista para tener un rendimiento superior, se le debe seleccionar sobre la base de la Morfoestructura, como la estatura, que permite mayor al- cance en altura; la masa muscular que se relaciona con la fuerza y potencia, y la masa grasa disminuida que permite menos peso de lastre y menos gasto energético.

Estos parámetros morfológicos son una parte esencial de la evaluación y selección de los deportistas, y que en nuestro país no existen dichas referencias. Estos patrones permiten determinar la evolución física del futbolista desde temprana edad, lo que ayuda enormemente en la intervención de los mismos, buscando mejoras morfológicas acordes con las necesidades de la especialidad y puesto de juego.

Como con otras actividades, el fútbol no es una ciencia, pero la ciencia puede ayudar a mejorar rendimiento deportivo. En éste estudio se presentan los resultados preliminares, que intentarán determinar las características morfológicas de los futbolistas chilenos de acuerdo a sus posiciones de juego, que permitan obtener referencias para elegir a los jugadores de acuerdo a su perfil antropométrico.

\footnotetext{
* Escuela de Nutrición y Dietética, Facultad de Medicina, Universidad Mayor, Chile.

${ }^{*}$ Laboratorio de Motricidad Humana, Escuela de Educación Física, Pontificia Universidad Católica de Valparaíso, Valparaíso, Chile.

${ }^{* * * *}$ Departamento de Medicina Aplicada a los Deportes. Club Atlético River Plate, Buenos Aires, Argentina.
} 


\section{MATERIAL Y METODO}

Se evaluó antropométricamente, a un total de 406 futbolistas profesionales, pertenecientes a 15 clubes chilenos, de los cuales 12 pertenecen a Primera División y 3 clubes a Primera B (Tabla I). Dentro de la muestra de jugadores, se logró evaluar a 48 Porteros, 124 Defensas centrales y laterales, 134 Volantes de Contención y de salida, y 93 Delanteros netos.

La evaluación antropométrica se realizó en las primeras horas de la mañana, previo a los entrenamientos, con la menor ropa posible(con pantalón corto) para facilitar el marcaje y el proceso evaluativo, después del vaciado urinario, en los mismos lugares de entrenamiento o estadio de cada equipo. Se midió bajo el protocolo de marcaje que recomienda la International Society for the Advancement of Kineatropometry (ISAK) y de evaluación descritos por Norton \& Olds (1996) y Drinkwater (1984), para el procedimiento de medición completa de 25 variables.

Para la aplicación del método antropométrico se utilizó el Kit Gaucho Pro "Mercosur", fabricado en Argentina bajo licencia de Rosscraft Canadá para su comercialización en el Continente Americano (excepto EE.UU, Canadá y México. El kit antropométrico está compuesto por los instrumentos y calibres: Campbell 20 (caliper largo), Campbell 10 (caliper corto), segmómetro retráctil, escuadra metálica, calibrador de pliegues cutáneos plástico o plicómetro, cinta métrica metálica, un estadiómetro portátil y una balanza Digital Tanita para el peso con precisión de $100 \mathrm{~g}$.

La recolección de los datos es facilitada por un asistente, quien ayuda al evaluador a registrar las variables. Dicho asistente se encuentra entrenado en las técnicas de medición, lo que permitió un trabajo fluido y rápido, de acuerdo al número de sujetos y al poco tiempo disponible, además aseguraba la secuencia correcta de los lugares de medición.

Se utilizó una ficha de recolección de datos o proforma electrónica, la cual fue diseñada con la intención de mejorar la rapidez de la medición, con un orden que va desde superior a inferior de la posición anatómica. Las medidas corresponden a 3 variables básicas, peso, estatura de pie y estatura sentado, 6 diámetros óseos, 10 perímetros o contornos axiales y apendiculares y 6 pliegues cutáneos.

Se determinó la forma corporal de los sujetos a través del método del Somatotipo de Carter (Carter \& Heath, 1990; Carter, 2002), los Z score del Phantom a través de las fórmulas de Ross (Ross \& Wilson de 1974) y la masa grasa a través de las fórmulas de Kerr (Kerr, 1988).
Se establecieron diferencias estadísticas a través de una prueba t entre las variables evaluadas y obtenidas como resultados, considerando un valor significativo de $\mathrm{p}<0,01$.

Tabla I. equipos y número de sujetos evaluados en el estudio.

\begin{tabular}{lr}
\hline EQUIPOS PROFESIONALES & N \\
\hline PRIMERA DIVISION & 30 \\
Audax Italiano & 18 \\
Cobreloa & 17 \\
Cobresal & 22 \\
Iquique & 28 \\
Nublense & 27 \\
O'Higgins & 31 \\
Palestino & 27 \\
Santiago Wanderers & 27 \\
Unión Española & 25 \\
Unión La Calera & 45 \\
Universidad Católica & 29 \\
Universidad de Chile & \\
PRIMERA B & 27 \\
La Serena & 27 \\
Santiago Morning & 26 \\
Unión San Felipe & $\mathbf{4 0 6}$ \\
\hline TOTAL
\end{tabular}

\section{RESULTADOS}

De los cuatro grupos según la posición de juego, se destaca una significancia estadística en el mayor peso de los Porteros, lo que coincide con la mayor estatura promedio de ese puesto de juego y por lo tanto del mayor IMC (Tabla II). También se aprecian diferencias significativas en la sumatoria de 6 pliegues, presentando el mayor valor promedio en comparación a las otras posiciones de juego.

A través de los pliegues cutáneos se estimó la masa grasa, donde en los porteros alcanza los $18 \mathrm{~kg}$, en los defensas, $16,2 \mathrm{~kg}$, en los volantes $14,9 \mathrm{~kg}$ y en los delanteros 15,4 $\mathrm{kg}$. Según la clasificación del laboratorio Biosystem de Argentina, todos los grupos se encuentran en la clasificación de "aceptable". Tampoco hay diferencias significativas en una prueba t, entre Defensas, Volantes y Delanteros en las medidas antropométricas básicas y masa grasa.

A continuación se presentan las medias de cada una de las variables antropométricas evaluadas, esto con la intención de que cada antropometrista, calcule y estime la composición corporal de acuerdo a las formulas y métodos que estime convenientes. Además se agregan para cada variable, los valores Z Score de acuerdo a las medidas del método Phantom, que permite comparar las medidas de acuerdo éste promedio asexuado humano. 
Tabla II. Medias y desvíos estándar de las variables básicas, IMC y Sumatoria de 6 Pliegues por posición de juego.

\begin{tabular}{|c|c|c|c|c|c|c|c|c|c|c|}
\hline \multirow[t]{2}{*}{\begin{tabular}{|l|} 
Posición \\
\end{tabular}} & \multicolumn{2}{|c|}{ Edad } & \multicolumn{2}{|c|}{ Peso } & \multicolumn{2}{|c|}{ Talla } & \multicolumn{2}{|c|}{ IMC } & \multicolumn{2}{|r|}{6 pliegues } \\
\hline & $\overline{\mathrm{X}}$ & D.E. & $\overline{\mathrm{X}}$ & D.E & $\overline{\mathrm{X}}$ & D.E & $\overline{\mathrm{X}}$ & D.E & $\overline{\mathrm{X}}$ & D.E \\
\hline Porteros & 25,1 & $\pm 5,5$ & (*) 81,4 & $\pm 5,9$ & $(*) 181,2$ & $\pm 3,8$ & 24,7 & $\pm 1,5$ & $(*) 58,9$ & $\pm 12,0$ \\
\hline Defensas & 25,3 & $\pm 4,8$ & 77,1 & $\pm 6,2$ & 178,1 & $\pm 5,5$ & 24,2 & $\pm 1,4$ & 51,3 & $\pm 13,6$ \\
\hline Volantes & 25,2 & $\pm 4,7$ & (**) 71,7 & $\pm 6,0$ & $(* *) 172,9$ & $\pm 5,6$ & 23,9 & $\pm 1,7$ & 50,5 & $\pm 11,6$ \\
\hline Delanteros & 23,5 & $\pm 4,1$ & 75,2 & $\pm 7,2$ & 176,6 & $\pm 6,1$ & 24,0 & $\pm 1,6$ & 48,8 & $\pm 11,5$ \\
\hline
\end{tabular}

(*) Valor significativamente más alto que los demás grupos para una prueba t con un valor de $\mathrm{p}<0,01$.

$(* *)$ Valor significativamente más bajo que los demás grupos para una prueba t con un valor de $\mathrm{p}<0,01$.

En la Tabla III, se aprecia que el diámetro anteroposterior del tórax presenta un Valor $\mathrm{Z}$ positivo, lo que se relaciona con la estaturapromedio de éstos sujetos. De manera opuesta el diámetro Bi-iliocrestideo presenta un Valor $\mathrm{Z}$ negativo, es decir que esta medida es más baja que el promedio de Phantom. Esto coincide con el perímetro de caderas (Trocantereo), que también presenta valores $\mathrm{Z}$ negativos.

Respecto de los perímetros, el de cabeza es el valor Z negativo más bajo. Los perímetros de los miembros superiores, son altos de acuerdo al Phantom y de los miembros inferiores, se encuentran apenas sobre la media Phantom. Se destaca que todos los valores $\mathrm{Z}$ de los pliegues cutáneos son negativos, pero no alcanzan a ser lo suficientemente bajos de acuerdo al profesionalismo en el que estos jugadores se desenvuelven.
En el Somatotipo no hay diferencias significativas en los valores de endomorfismo (Grasa relativa), Mesomorfismo (Musculo relativo), ni ectomorfismo (Linealidad relativa), que se refleja también en los valores de coordenadas x e y para la somatocarta (Tabla IV).

La clasificación promedio de los futbolistas chilenos es Mesomorfico balanceado, donde predomina el músculo relativo. El Endomorfismo se caracteriza como "Baja adiposidad relativa; poca grasa subcutánea; contornos musculares y óseos visibles". El Mesomorfismo como "Alto desarrollo músculo-esquelético relativo; diámetros óseos grandes; músculos de gran volumen; articulaciones grandes" y el Ectomorfismo como "Gran volumen por unidad de altura; extremidades relativamente voluminosas". La representación de los valores del Somatotipo en la somatocarta, muestran la similitud señalada anteriormente (Fig. 1).

Tabla III. medias y valores Z Score Phantom de las variables antropométricas medidas

\begin{tabular}{|c|c|c|c|c|c|c|c|c|}
\hline \multirow[b]{2}{*}{ VARIABLES ANTROPOMETRICAS } & \multicolumn{2}{|c|}{ PORTEROS } & \multicolumn{2}{|c|}{ DEFENSAS } & \multicolumn{2}{|c|}{ VOLANTES } & \multicolumn{2}{|c|}{ DELANTEROS } \\
\hline & \multicolumn{2}{|c|}{ Media Z Score } & \multicolumn{2}{|c|}{ Media Z Score } & \multicolumn{2}{|c|}{ Media Z Score } & \multicolumn{2}{|c|}{ Media Z Score } \\
\hline \multicolumn{9}{|l|}{ Diametros } \\
\hline Biacromial & 41,9 & 0,7 & 41,0 & 0,6 & 40,1 & 0,7 & 40,9 & 0,7 \\
\hline Tórax Transverso & 30,7 & 0,5 & 29,9 & 0,4 & 29,2 & 0,5 & 30,0 & 0,6 \\
\hline Tórax Anteroposterior & 20,6 & 1,3 & 20,3 & 1,4 & 20,7 & 2,1 & 20,1 & 1,4 \\
\hline Bi-iliocrestídeo & 28,7 & $-1,1$ & 28,4 & $-1,0$ & 27,7 & $-0,9$ & 27,9 & $-1,1$ \\
\hline Humeral (biepicondilar) & 7,2 & 0,9 & 7,1 & 0,8 & 6,9 & 1,0 & 7,0 & 0,6 \\
\hline Femoral (biepicondilar) & 10,3 & 0,3 & 10,1 & 0,3 & 9,9 & 0,5 & 10,0 & 0,2 \\
\hline \multicolumn{9}{|l|}{ Perimetros } \\
\hline Cabeza & 57,1 & $-1,6$ & 56,8 & $-1,2$ & 56,3 & $-0,4$ & 56,5 & $-1,0$ \\
\hline Brazo Relajado & 31,9 & 1,3 & 30,5 & 1,0 & 29,5 & 0,9 & 30,1 & 0,9 \\
\hline Brazo Flexionado en Tensión & 34,2 & 1,1 & 33,4 & 1,1 & 31,9 & 0,9 & 32,5 & 0,8 \\
\hline Antebrazo & 28,2 & 1,0 & 27,1 & 0,5 & 26,5 & 0,7 & 27,0 & 0,7 \\
\hline Tórax Mesoesternal & 100,7 & 1,3 & 97,9 & 1,1 & 95,3 & 1,1 & 96,9 & 1,1 \\
\hline Cintura (mínima) & 82,6 & 1,3 & 80,8 & 1,2 & 79,5 & 1,4 & 80,1 & 1,2 \\
\hline Caderas (máxima) & 98,8 & $-0,3$ & 97,4 & $-0,3$ & 95,0 & $-0,2$ & 96,4 & $-0,3$ \\
\hline Muslo (superior) & 59,6 & 0,0 & 58,9 & 0,1 & 57,2 & 0,1 & 58,1 & 0,0 \\
\hline Muslo (medial) & 55,2 & 0,4 & 55,0 & 0,4 & 53,8 & 0,1 & 54,3 & 0,2 \\
\hline Pantorrilla (máxima) & 38,4 & 0,4 & 38,0 & 0,5 & 37,1 & 0,5 & 37,8 & 0,5 \\
\hline \multicolumn{9}{|l|}{ Pliegues Cutáneos } \\
\hline Tríceps & 8,6 & $-1,6$ & 7,7 & $-1,8$ & 7,5 & $-1,8$ & 7,0 & $-1,9$ \\
\hline Subescapular & 9,5 & $-1,6$ & 8,6 & $-1,8$ & 8,7 & $-1,7$ & 8,5 & $-1,8$ \\
\hline Supraespinal & 8,8 & $-1,6$ & 7,7 & $-1,8$ & 7,4 & $-1,8$ & 7,2 & $-1,9$ \\
\hline Abdominal & 16,3 & $-1,3$ & 13,5 & $-1,6$ & 13,2 & $-1,6$ & 12,8 & $-1,7$ \\
\hline Muslo (medial) & 9,4 & $-2,2$ & 8,6 & $-2,3$ & 8,7 & $-2,2$ & 8,2 & $-2,3$ \\
\hline Pantorrilla & 6,3 & $-2,2$ & 5,3 & $-2,3$ & 5,2 & $-2,3$ & 5,1 & $-2,4$ \\
\hline
\end{tabular}


Tabla IV. medias de cada componente del Somatotipo y de las coordenadas x e y.

\begin{tabular}{llllll}
\hline Posición & Endo & Meso & Ecto & X & Y \\
\hline Arqueros & 2,53 & 5,49 & 2,05 & $-0,45$ & 6,25 \\
Defensas & 2,25 & 5,40 & 2,10 & $-0,15$ & 6,43 \\
Volantes & 2,20 & 5,51 & 1,94 & $-0,33$ & 6,79 \\
Delanteros & 2,13 & 5,32 & 2,10 & $-0,33$ & 6,49 \\
\hline
\end{tabular}

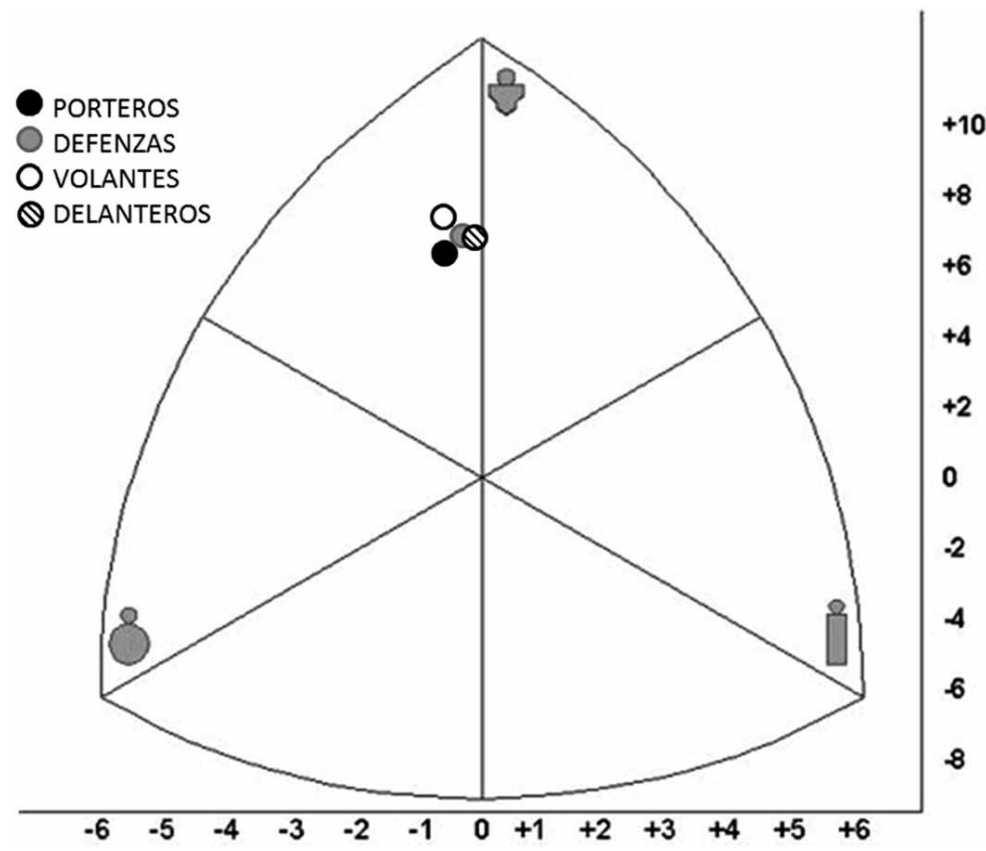

Fig. 1. Somatotipo de los cuatro grupos de futbolistas.

\section{DISCUSIÓN}

Variables básicas. Los porteros presentan diferencias significativas respecto de los otros grupos en el peso corporal, estatura y la sumatoria de 6 pliegues, lo que hace referencia al peso en masa grasa, la cual está determinada por el gasto energético que se realiza en los entrenamiento y competencia, el cual en éste grupo es menor. Estos jugadores con mayor movilidad, pueden llegar a recorrer entre $10 \mathrm{a} 11 \mathrm{~km}$ por partido (Barros et al., 2007; Bangsbo \& Michalsik, 2002; Tumilty, 1993; Di Salvo et al., 2006). Un estudio de Mohr et al. (2003), hecho en 80 jugadores de la Champions League, señala que los metros recorridos no son continuos sino intermitentes, donde casi 40 min del partido se hacen caminando y no más de 5 min se realizan a alta intensidad, por lo que el gasto energético y la movilidad de los porteros en mucho menor. Parece ser que mientras más defensiva es la posición de juego, menos intensa es la actividad física que se realiza.

En la actualidad priman los jugadores especialistas en una posición de juego y que deben cumplir ciertas características. Es coherente que los jugadores que disputen balones aéreos, tengan mayor estatura para mejorar el éxito en la posesión del balón, por lo que los defensas deben tener una estatura mayor y significativa frente a los otros puestos. En éste estudio los Defensas son más altos que los Volantes $(\mathrm{p}<0,00)$, pero no de los Delanteros $(\mathrm{p}<0,02)$. Esto es coherente y pone en igualdad de condición al ataque y la defensa en el juego aéreo, pero ésta estatura (Tabla II), es menor en comparación a defensas Daneses quienes tienen una estatura de 189+0,04 cm (Reilly et al., 2000a, 2000b), más de $10 \mathrm{~cm}$ de diferencia, lo que nos pone en desventaja en eventos internacionales. Es necesario destacar que la estatura es el factor de talento en el futbol más importante con un valor $\mathrm{r}=0,85$.

Método Phantom. Al comparar las variables con el método Phantom, los jugadores tienen valores " $Z$ " coherentes con el deporte, excepto en la masa grasa, donde a pesar de ser valores negativos, la sumatoria de 6 pliegues, indica valores "aceptables", por lo que es necesario disminuir éste componente, lo que permita mejorar su relación peso/potencia, donde el peso es importante, como en los saltos y aceleraciones.

En este estudio los Volantes presentan menor peso y estatura, al igual que en otros estudios (Sporis et al., 2009; Cossio-Bolanos et al., 2012 ), donde además recorren mayor cantidad de metros. La gran movilidad de los volantes hace necesario un perfil antropométrico eficiente, bajo peso y baja masa grasa.

Otra debilidad que se aprecia en el Phantom, es que los perímetros de los miembros inferiores se encuentran muy cercanos a la media, lo que podría indicar una masa muscular "normal" que no favorece la potencia o la fuerza (Wong et al., 2010), las cuales son las capacidades fundamentales para realizar las acciones deportivas que priman en el futbol, como son saltar, patear, correr con velocidad y realizar cambios de ritmo (Stolen et al.).

Método de Somatotipo. El somatotipo de los jugadores muestra un equilibrio entre el Endomorfismo y el Ectomofismo. El Endomorfismo bajo es favorable lo que significa una menor forma corporal relacionada con la masa grasa, y el Ectomorfismo alto denota una robustez ósea que puede afectar negativamente el peso corporal, pero que puede favorecer el desarrollo de la masa muscular y evi- 
tar lesiones óseas o por sobrecarga. Esta baja linealidad da una apariencia "gruesa y robusta" más típica de los jugadores de rugby, pero con una estatura menor. Estos hallazgos se contrastan con los encontrados en futbolistas de la India (Bandyopadhyay, 2007), donde el Ectomorfismo es mayor y por lo tanto la robustez ósea menor.

La clasificación del somatotipo coincide con la de otros futbolistas europeos (Reilly et al., 2000b), donde prima el mesomorfismo seguido del ectomorfismo. A pesar de éstas similitudes el Mesomorfismo varía en distintos grupos de jugadores de elite (Hazir, 2010), por ejemplo en Sudamerica es 2,2-5,4-2,2 (Rienzi et al., 2000), Europa es 2,4-4,8-2,3 (Casajús, 2001), Asia es 2,70-4,94-2,95 (Rahmawati et al., 2007), y África es 2,2-5,4-2,9 (Mathur et al., 1985). Esto demuestra que desde el punto de vista del somatotipo, los futbolistas chilenos tienen una forma corporal similar a la de otros equipos internacionales.

\section{CONCLUSIÓN}

A la luz de nuestros resultados, la antropometría puede concebirse como un sistema económico y fácil de aplicar, que permite la monitorización y la evaluación de los futbolistas con vista a la planificación y formación individual de los jugadores (Alburquerque et al., 2005).
Existen diferencias en algunas variables antropométricas básicas por posición de juego, que nos ponen en desventaja frente a otros jugadores internacionales, como la estatura.

No hay diferencias en el Phantom ni en el somatotipo por posición de juego, es decir tienen variables relativamente homogéneas, lo que se provoca al comparar grupos con una gran cantidad de sujetos que los componen. Esto podría hacernos pensar que un jugador puede desempeñarse en cualquier posición de juego, pero hemos visto que la estatura, el peso y la masa grasa son distintas por cada posición, por lo que cada jugador tiene su función y sus propias características en las que los entrenadores y preparadores deben fijarse al momento de ubicar a cada jugador en su función específica, ya que estas características influirán en su desempeño físico en el campo de juego.

\section{AGRADECIMIENTOS}

A los preparadores físicos y entrenadores de los equipos evaluados, por su disposición y tiempo para que el estudio se realizara, a los colaboradores y evaluadores que favorecieron el registro de los datos en el trabajo de campo y Escuela de Nutrición y Dietética, Facultad de Medicina, Universidad Mayor, Chile.

JORQUERA, A. C.; RODRÍGUEZ, R. F.; TORREALBA, V. M. I.; CAMPOS, S. J.; GRACIA, L. N. \& HOLWAY, F. Anthropometric characteristics of Chilean professional football players. Int. J. Morphol., 31(2):609-614, 2013.

SUMMARY: The trend in the formation in football, determines that the anthropometric variables are important when defining among other things, the most suitable playing position. These morphological parameters are an essential part of the evaluation and selection of athletes. These patterns can determine the physical evolution of football from an early age, which helps tremendously in the operation thereof. Anthropometrically, a total of 406 professional footballers from 15 Chilean clubs were evaluated, of which 12 belong to the first division clubs and 3 to First B. There are differences in some basic anthropometric variables by playing position, which places us at a disadvantage compared to other international players, such as height. No differences in the Phantom or the somatotype by playing position, i.e. variables are relatively homogeneous. This could suggest that a player can perform at any position in the game, but we have seen that the height, weight and fat mass are different for each position, so that each player has their own characteristics and their role in the coaches and trainers should be fixed at the time of placing each player on their specific function.

KEY WORDS: Anthropometry; Soccer; Field position.

\section{REFERENCIAS BIBLIOGRÁFICAS}

Alburquerque, F.; Sánchez, F.; Prieto, J.; López, N., \& Santos, M. Kinanthropometric assessment of a football team over one season. Eur. J. Anat., 9(1):17-22, 2005.

Bandyopadhyay, A. Anthropometry and body composition in soccer and volleyball players in West Bengal, India. J. Physiol. Anthropol., 26(4):501-5, 2007.
Bangsbo, J. \& Michalsik, L. Assessment of the physiological capacity of elite soccer players. In: Science and Football IV. Spinks, W.; Reilly, T. \& Murphy, A. (Eds.). London, Routledge, 2002. pp.53-62.

Barros, R.; Misuta, M.; Menezes, R.; Figueroa, P.; Moura, F.; Cunha, S.; et al. Analysis of the distances covered by first 
division Brazilian soccer players obtained with an automatic tracking method. J. Sports Sci. Med., 6(2):233-42, 2007.

Carter, J. E. L. The Heath-Carter Somatotype method. San Diego, San Diego State University Syllabus Service, 2002.

Carter, J. E. L. \& Heath, B. H. Somatotyping - Development and Applications. Cambridge, Cambridge University Press, 1990.

Casajús, J. A. Seasonal variation in fitness variables in professional soccer players. J. Sports Med. Phys. Fitness, 41(4):463-9, 2001.

Cossio-Bolanos, M.; Portella, D.; Hespanhol, J. E.; Fraser, N. \& Arruda, M. Body Size and Composition of the Elite Peruvian Soccer Player. JEPonline, 15(3):30-8, 2012.

Dey, S. K.; Kar, N. \& Debray, P. Anthropometric, motor ability and physiological Profiles of Indian national club footballers: A comparative study. S. Afr. J. Res. Sport Ph., 32(1):43-56, 2010 .

Di Salvo, V.; Collins, A.; McNeill, B. \& Cardinale, M. Validation of Prozone $\circledR$ : A new video-based performance analysis system. Int. J. Perform. Anal. Sport, 6(1):108-19, 2006.

Drinkwater, D. T. An anatomically deroved method for the anthropometric estimation of human body composition. Ph.D. Thesis, British Columbia, Simon Fraser University, 1984.

Hazir, T. Physical characteristics and somatotype of soccer players according to playing level and position. J. Hum. Kinetics, 26:83-95, 2010.

Kerr, D. A. An anthropometric method for the fractionation of skin, adipose, muscle, bone and residual tissue masses in males and females age 6 to 77 years. M.Sc. Thesis. British Columbia, Simon Fraser University, 1988.

Mathur, D. N.; Toriola, A. L. \& Igbokwe, N. U. Somatotypes of Nigerian athletes of several sports. Br. J. Sports Med., 19(4):219-20, 1985.

Mohr, M.; Krustrup, P. \& Bangsbo, J. Match performance of highstandard soccer players with special reference to development of fatigue. J. Sports Sci., 21(7):519-28, 2003.

Norton, K. \& Olds, T. Antropometrica. Marrickville, Ed. Southwood Press, 1996.

Rahmawati, N. T.; Budiharjo, S. \& Ashizawa, K. Somatotypes of young male athletes and non-athlete students in Yogyakarta, Indonesia. Antropol. Sci., 115(1):1-7, 2007.

Reilly, T.; Bangsbo, J. \& Franks, A. Anthropometric and physiological predispositions for elite soccer. J. Sports Sci., 18(9):669-83, 2000a.

Reilly, T.; Williams, A. M.; Nevill, A. \& Franks, A. A multidisciplinary approach to talent identification in soccer. $J$. Sport Sci., 18(9):695-702, 2000b.

Rienzi, E.; Drust, B.; Reilly, T.; Carter, J. E. \& Martin, A. Investigation of anthropometric and work-rate profiles of elite South American international soccer players. J. Sports Med. Phys. Fitness, 40(2):162-9, 2000.

Ross W. D. \& Wilson N. C. A strategem for proportional growth assessment. Acta Paediatr. Belg., 28 suppl:169-82, 1974.

Sporis, G.; Jukic, I.; Ostojic, S. M. \& Milanovic, D. Fitness profiling in soccer: physical and physiologic characteristics of elite players. J. Strength Cond. Res., 23(7):1947-53, 2009.

Stolen, T.; Chamari, K.; Castagna, C. \& Wisloff, U. Physiology of soccer: an update. Sports Med., 35(6):501-36, 2005.

Tumilty, D. Physiological characteristics of elite soccer players. Sports Med., 16(2):80-96, 1993.

Wong, P. L.; Chaouachi, A.; Chamari, K.; Dellal, A. \& Wisloff, U. Effect of preseason concurrent muscular strength and highintensity interval training in professional soccer players. $J$. Strength Cond. Res., 24(3):653-60, 2010.

Dirección para correspondencia:

Fernando Rodríguez Rodríguez

Avda. El Bosque 1290

Casilla 4059

Valparaíso

Pontificia Universidad Católica de Valparaíso

CHILE

E-mail: fernando.rodriguez@ucv.cl

Recibido : 26-02-2013

Aceptado: 17-04-2013 